02,12

\title{
Анизотропия эффекта Холла в квазидвумерном электронно-легированном сверхпроводнике $\mathrm{Nd}_{2-x} \mathrm{Ce}_{x} \mathrm{CuO}_{4+\delta}$
}

\author{
(C) А.С. Клепикова ${ }^{1}$, Т.Б. Чарикова ${ }^{1,2}$, Н.Г. Шелушинина ${ }^{1}$, Д.С. Петухов ${ }^{1}$, А.А. Иванов ${ }^{3}$ \\ ${ }^{1}$ Институт фризики металлов им. М.Н. Михеева УрО РАН, \\ Екатеринбург, Россия \\ ${ }^{2}$ Уральский федеральный университет им. Б.Н. Ельцина, \\ Екатеринбург, Россия \\ ${ }^{3}$ Национальный исследовательский ядерный университет „МИФИ“, \\ Москва, Россия \\ E-mail: klepikova@imp.uran.ru
}

\begin{abstract}
Представлены результаты исследования температурных зависимостей эффекта Холла электроннолегированного сверхпроводника $\mathrm{Nd}_{2-x} \mathrm{Ce}_{x} \mathrm{CuO}_{4+\delta}$ на границе квантового фазового перехода антиферромагнетик - сверхпроводник $(0.135 \leq x \leq 0.15)$ в проводящих плоскостях $\mathrm{CuO}_{2}$ и в направлении, перпендикулярном плоскостям $\mathrm{CuO}_{2}$. Экспериментально обнаружено, что величина коэффициента Холла между проводящими плоскостями на два порядка больше, чем в проводящих плоскостях во всем диапазоне температур, что обусловлено некогерентным характером переноса носителей заряда в направлении оси $c$.
\end{abstract}

Работа выполнена в рамках государственного задания по теме „Электрон“ АААА-А18-118020190098-5 и проекту № 18-10-2-6 Программы УрО РАН при поддержке РФФИ (грант № 18-02-00192).

DOI: 10.21883/FTT.2018.11.46650.14NN

\section{1. Введение}

Соединение $\mathrm{Nd}_{2-x} \mathrm{Ce}_{x} \mathrm{CuO}_{4+\delta}$ - это сверхпроводник с электронным типом проводимости, имеет объемноцентрированную кристаллическую решетку и соответствует тетрагональной $T^{\prime}$-фазе. Параметры решетки: $a=b=0.394 \mathrm{~nm}, c=1.208 \mathrm{~nm}$. В результате оптимального легирования $(x=0.15)$ и отжига $(\delta \rightarrow 0)$ кристаллическая структура представляет собой набор проводящих плоскостей $\mathrm{CuO}_{2}$, отделенных расстоянием $0.6 \mathrm{~nm}$ в направлении оси $c$ [1]. Соединение обладает ярко выраженными двумерными свойствами - в том числе, квазидвумерным характером переноса носителей заряда.

Исследованное соединение $\mathrm{Nd}_{2-x} \mathrm{Ce}_{x} \mathrm{CuO}_{4+\delta}$ характеризуется способностью обратимо поглощать и выделять кислород. Оптимальное содержание кислорода в элементарной ячейке, а, следовательно, и максимальная $T_{c}$ наблюдается, когда $\delta \rightarrow 0$. Проблема анизотропии удельного сопротивления в нормальном состоянии систем оксидов меди давно привлекает внимание исследователей. Сопротивление в разных направлениях отличается не только величиной, но и характером температурной зависимости. Изучение металлического поведения $\left(d \rho_{a b} / d T>0\right)$ в проводящих $\mathrm{CuO}_{2}-$ плоскостях и неметаллического поведения $\left(d \rho_{c} / d T<0\right)$ в $c$-направлении являются ключевым моментом в теоретическом и экспериментальном анализе системы носителей зарядов купратных высокотемпературных сверхпроводников (ВТСП) [2,3].

Неметаллическая проводимость вдоль оси $c$ в сочетании с металлической проводимостью в $\mathrm{CuO}_{2}-$ плоскости при сильной анизотропии проводящих свойств неоднократно наблюдалась в дырочных [4] и электронно- легированных [5] ВТСП, что является экспериментальным свидетельством квазидвумерности оксидных систем. В данной работе проведено исследование сопротивления Холла, $\rho_{x y}$, в плоскости $\mathrm{CuO}_{2}$ и между плоскостями $\mathrm{CuO}_{2}$ в электронно-легированном ВТСП $\mathrm{Nd}_{2-x} \mathrm{Ce}_{x} \mathrm{CuO}_{4+\delta}$ в области перехода от фазы сосуществования антиферромагнитного (АФМ) и сверхпроводящего (СП) упорядочения в сверхпроводящую фазу с целью анализа анизотропии переноса носителей заряда.

\section{2. Методика эксперимента}

Методом импульсного лазерного осаждения [6,7] были синтезированы эпитаксиальные пленки $\mathrm{Nd}_{2-x} \mathrm{Ce}_{x} \mathrm{CuO}_{4+\delta} / \mathrm{SrTiO}_{3} \quad$ с $\quad x=0.135,0.145 \quad$ и 0.15 трех типов:

1. Ориентация пленки (001) - $c$-ось решетки $\mathrm{Nd}_{2-x} \mathrm{Ce}_{x} \mathrm{CuO}_{4+\delta}$ перпендикулярна плоскости подложки $\mathrm{SrTiO}_{3}$.

2. Ориентация пленки $(1 \overline{1} 0)-$ ось $c$ решетки $\mathrm{Nd}_{2-x} \mathrm{Ce}_{x} \mathrm{CuO}_{4+\delta}$ направлена вдоль длинной стороны подложки $\mathrm{SrTiO}_{3}$.

3. Ориентация пленки $(1 \overline{1} 0)-$ ось $c$ решетки $\mathrm{Nd}_{2-x} \mathrm{Ce}_{x} \mathrm{CuO}_{4+\delta}$ направлена вдоль короткой стороны подложки $\mathrm{SrTiO}_{3}$. Такая конфигурация пленки была выбрана для измерения температурной зависимости эффекта Холла между плоскостями $\mathrm{CuO}_{2}$.

В процессе импульсного лазерного осаждения был использован эксимерный лазер $\mathrm{KrF}$ с длиной волны $248 \mathrm{~nm}$, с энергией $80 \mathrm{~mJ} / \mathrm{imp}$ и плотностью энергии на поверхности мишени $1.5 \mathrm{~J} / \mathrm{cm}^{2}$. Длительность импульса 
составляла $15 \mathrm{~ns}$, частота следования импульсов от 5 до $20 \mathrm{~Hz}$. Далее синтезированные пленки были подвергнуты термообработке (отжигу) при различных условиях для получения образцов с разным содержанием кислорода. Рентгеноструктурный анализ (Со- $K_{\alpha}$ излучение) показал, что все пленки имели высокое качество и являлись монокристаллическими. Для измерений были отобраны пленки $\mathrm{Nd}_{2-x} \mathrm{Ce}_{x} \mathrm{CuO}_{4+\delta} / \mathrm{SrTiO}_{3}$ с оптимальным отжигом - это подразумевает образцы с максимальным значением температуры сверхпроводящего перехода для данного содержания церия $(x)$. Условия оптимального отжига были следующие:

- для состава $x=0.15\left(T_{c}^{\text {onset }}=23.5 \mathrm{~K}, T_{c}=22 \mathrm{~K}\right)-$ $t=60 \mathrm{~min}, T=780^{\circ} \mathrm{C}, p=10^{-5} \mathrm{~mm} \mathrm{Hg}$;

- для состава $x=0.145 \quad\left(T_{c}^{\text {onset }}=15.7 \mathrm{~K}\right.$, $\left.T_{c}=10.7 \mathrm{~K}\right)-t=60 \mathrm{~min}, T=600^{\circ} \mathrm{C} p=10^{-5} \mathrm{~mm} \mathrm{Hg}$; - для состава $x=0.135 \quad\left(T_{c}^{\text {onset }}=13.7 \mathrm{~K}\right.$, $\left.T_{c}=9.6 \mathrm{~K}\right)-t=60 \mathrm{~min} ., \quad T=600^{\circ} \mathrm{C}, p=10^{-5} \mathrm{~mm} \mathrm{Hg}$.

Толщина пленок составляла $d=140-520 \mathrm{~nm}$.

Температурные зависимости продольного сопротивления $\rho_{x x}(T)$ и сопротивления Холла $\rho_{x y}(T)$ для всех исследованных типов пленок $\mathrm{Nd}_{2-x} \mathrm{Ce}_{x} \mathrm{CuO}_{4+\delta} / \mathrm{SrTiO}_{3}$ были проведены на установке Quantum Design PPMS 9 и в соленоиде „Oxford Instruments“(ЦКП „Испытательный центр нанотехнологий и перспективных материалов“ ИФМ УрО РАН). Электрическое поле было приложено всегда параллельно плоскости подложки $\mathrm{SrTiO}_{3}$. Внешнее магнитное поле $B$ всегда было направлено перпендикулярно плоскости подложки $\mathrm{SrTiO}_{3}$. В зависимости от типа измеряемых образцов мы получили возможность измерить температурные зависимости сопротивления в проводящих плоскостях $\mathrm{CuO}_{2}$ и между плоскостями (вдоль оси $c$ ). В скрещенных электрическом и магнитном полях на разных типах образцов были измерены температурные зависимости сопротивления Холла: в проводящих плоскостях $\mathrm{CuO}_{2}$ и между плоскостями (вдоль оси $c$ ).

\section{3. Результаты и обсуждение}

Проведенное в [8] исследование температурных зависимостей сопротивления $\rho_{a b}(T)$ и $\rho_{c}(T)$ в стехиометрических (оптимально отожженных) соединениях $\mathrm{Nd}_{2-x} \mathrm{Ce}_{x} \mathrm{CuO}_{4+\delta}$ на границе перехода АФМ-СП $(0.135 \leq x \leq 0.15)$ указывает на наличие некогерентного переноса носителей заряда в направлении оси $c$ некогерентное туннелирование между проводящими плоскостями. Удельное сопротивление $\rho_{c}$ в направлении оси $c$, поперек проводящих плоскостей $\mathrm{CuO}_{2}$, является большим по сравнению с сопротивлением $\rho_{a b}$ в проводящих плоскостях и имеет неметаллическую температурную зависимость в исследованных образцах. Таким образом имеет место квазидвумерный характер переноса носителей заряда в пленках с $0.135 \leq x \leq 0.15$ вблизи квантового фазового перехода АФМ-СП.

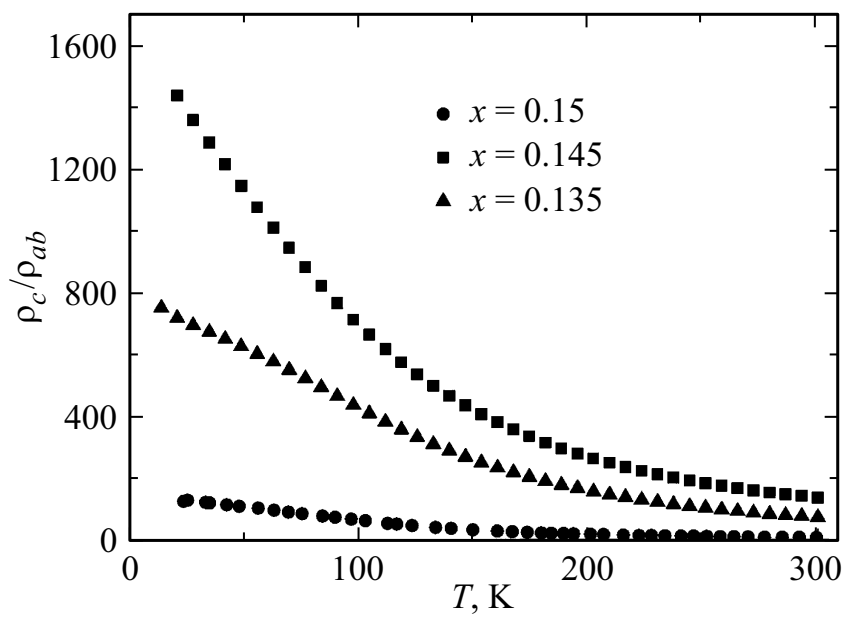

Рис. 1. Температурная зависимость коэффициента анизотропии сопротивления для оптимально отожженных пленок $\mathrm{Nd}_{2-x} \mathrm{Ce}_{x} \mathrm{CuO}_{4+\delta} / \mathrm{SrTiO}_{3}$.

На рис. 1 представлена температурная зависимость коэффициента анизотропии сопротивления в проводящих плоскостях $\mathrm{CuO}_{2}$ и в направлении, перпендикулярном этим плоскостям. Из рис. 1 видно, что коэффициент анизотропии удельного сопротивления велик даже при комнатной температуре: $\rho_{c} / \rho_{a b} \approx 10-10^{2}$ для всех исследованных пленок. Этот параметр существенно возрастает с понижением температуры, достигая значений $\rho_{c} / \rho_{a b} \sim 10^{3}$ для соединений с $x=0.145$ и 0.135 и $\rho_{c} / \rho_{a b} \approx 10^{2}$ для оптимально легированного соединения с $x=0.15$ из-за резкого роста удельного сопротивления $\rho_{c}$ при низких температурах.

Сильная анизотропия сопротивления в слоистой системе $\mathrm{Nd}_{2-x} \mathrm{Ce}_{x} \mathrm{O}_{4+\delta}$ может быть объяснена некогерентным переносом носителей заряда в направлении c $[9,10]$ при хорошей металлической проводимости в $\mathrm{CuO}_{2}$-плоскостях. В области нормального состояния, при температурах $T>T^{*}>T_{c}\left(T^{*}-\right.$ температура перехода в псевдощелевое состояние, $T_{c}$ - критическая температура сверхпроводящего перехода), когда сильные электронные корреляции не столь существенны и не приводят к возникновению фазы страйпового упорядочения, появлению волн зарядовой и спиновой плотности и к возникновению псевдощелевого состояния, эффектами электрон-электронного взаимодействия можно пренебречь [11]. Тогда если вероятность рассеяния носителя в плоскости, $\hbar / \tau$, много больше, чем интеграл переноса, $t_{c}$, между плоскостями, т. е. электрон испытывает много столкновений прежде, чем перейти в другую плоскость, то последовательные процессы туннелирования между плоскостями не коррелированы (некогерентный перенос - неметаллическая проводимость вдоль оси $c$ ).

Согласно $[9,10]$, в модели некогерентного переноса носителей заряда коэффициент диффузии квазидвумерной системы вдоль, и поперек, слоев описывается выра- 


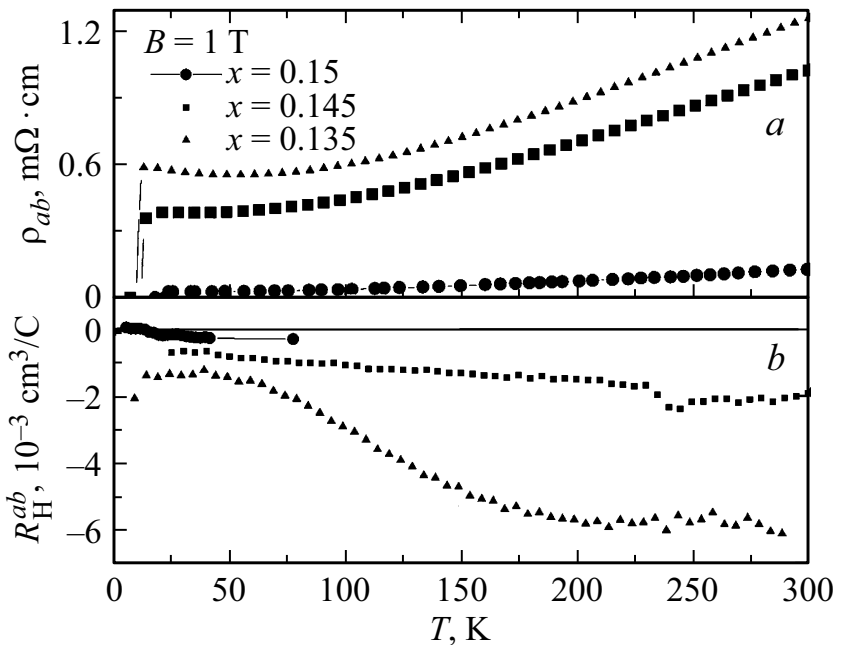

Рис. 2. Температурные зависимости удельного сопротивления в $\mathrm{CuO}_{2}$-плоскости, $\rho_{a b}(a)$ и коэффициента Холла при $B=1 \mathrm{~T}$ в $\mathrm{CuO}_{2}$-плоскости, $R_{H}^{a b}(b)$ в пленках $\mathrm{Nd}_{2-x} \mathrm{Ce}_{x} \mathrm{CuO}_{4+\delta} / \mathrm{SrTiO}_{3}$ для $0.135 \leq x \leq 0.15$ и оптимальном отжиге.

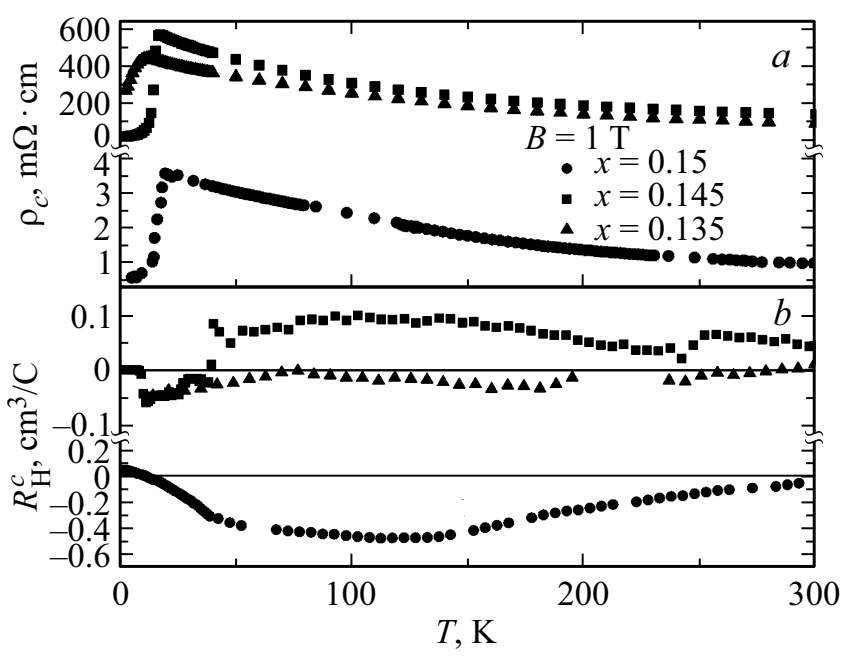

Рис. 3. Температурные зависимости удельного сопротивления вдоль оси $c, \rho_{c}(a)$ и коэффициента Холла при $B=1 \mathrm{~T}$ между плоскостями, $R_{H}^{c}(b)$ в пленках $\mathrm{Nd}_{2-x} \mathrm{Ce}_{x} \mathrm{CuO}_{4+\delta} / \mathrm{SrTiO}_{3}$ для $0.135 \leq x \leq 0.15$ и оптимальном отжиге.

жениями:

$$
D_{\|}=\frac{1}{2} \frac{l^{2}}{\tau} ; \quad D_{\perp}=\frac{1}{2} c^{2}\left(\frac{t_{c}}{\hbar}\right)^{2} \tau,
$$

где $l$ - длина свободного пробега, а $\tau-$ время релаксации носителей в $\mathrm{CuO}_{2}$-плоскости, $c=0.6 \mathrm{~nm}-$ расстояние между соседними $\mathrm{CuO}_{2}$-плоскостями.

Из (1) находим

$$
\frac{\rho_{c}}{\rho_{a b}}=\frac{D_{\|}}{D_{\perp}}=\left(\frac{l}{c}\right)^{2}\left(\frac{\hbar}{t_{c} \tau}\right)^{2},
$$

и $\rho_{c} / \rho_{a b} \gg 1$, так как $l / c \gg 1$ и в условиях некогерентного туннелирования $t_{c} \tau \ll \hbar$.
Температурная зависимость коэффициента Холла в пленках с ориентацией оси $c(001)$ и $(1 \overline{1} 0)$, в сопоставлении с поведением удельного сопротивления для тех же ориентаций, представлена на рис. 2 и 3 . Коэффициент Холла $R_{H}^{a b}$, соответствующий движению носителей в плоскости $\mathrm{CuO}_{2}$, отрицателен в температурном интервале $T=(1.8-300) \mathrm{K}$ для всех исследованных пленок, а его модуль увеличивается с ростом температуры (рис. $2, b$ ). Более того, $\left|R_{H}^{a b}\right|$ уменьшается с увеличением уровня легирования, и в рамках однозонной модели $\left(n=1 / e R_{H}\right)$ при $T=77 \mathrm{~K}$ находим:

$$
\begin{aligned}
& n_{x=0.135}=3.05 \cdot 10^{21} 1 / \mathrm{cm}^{3} ; \\
& n_{x=0.145}=6.25 \cdot 10^{21} 1 / \mathrm{cm}^{3} \\
& n_{x=0.15}=2.16 \cdot 10^{22} 1 / \mathrm{cm}^{3}
\end{aligned}
$$

Уменьшение $\left|R_{H}^{a b}\right|$ с увеличением уровня легирования сильнее, чем $1 / x$, указывает на дополнительный вклад носителей заряда второго типа (дырок) за счет реконструкции поверхности Ферми (см. работу [12] и ссылки в ней). Экспериментально обнаружено, что величина коэффициента Холла между проводящими плоскостями $\left|R_{H}^{c}\right|$ на два порядка больше, чем $\left|R_{H}^{a b}\right|$ в проводящих плоскостях во всем диапазоне температур (рис. $3, b)$.

В модели некогерентного переноса $[9,10]$ (см. также [13]) можно показать, что коэффициент Холла для движения электронов поперек проводящих плоскостей, $R_{H}^{c}$, не дает информации о концентрации носителей, а отношение коэффициентов $R_{H}^{c}$ и $R_{H}^{a b}$ описывается выражением:

$$
\frac{R_{H}^{c}}{R_{H}^{a b}}=\frac{1}{\omega_{c} \tau} \cdot \frac{\hbar}{t_{c} \tau} \cdot \frac{\rho_{a b}}{\rho_{c}}
$$

где $\omega_{c}=e B / m-$ циклотронная частота. При $B=1 \mathrm{~T}$ для исследованных образцов $\omega_{c} \tau \cong 10^{-3}$. Тогда, если $\frac{\rho_{a b}}{\rho_{c}} \cong\left(10^{-2}-10^{-3}\right)$ и $\frac{\hbar}{t_{c} \tau}($ см. оценки в $[8])$, то из (3) находим $R_{H}^{c} / R_{H}^{a b} \cong\left(10-10^{2}\right)$ в достаточно хорошем соответствии с экспериментальными данными.

\section{4. Заключение}

Впервые экспериментально измерено и исследовано поведение температурных зависимостей эффекта Холла электронно-легированного сверхпроводника $\mathrm{Nd}_{2-x} \mathrm{Ce}_{x} \mathrm{CuO}_{4+\delta}$ на границе квантового фазового перехода антиферромагнетик-сверхпроводник $(0.135 \leq x \leq 0.15)$ в проводящих плоскостях $\mathrm{CuO}_{2}$ и в направлении, перпендикулярном плоскостям $\mathrm{CuO}_{2}$, на монокристаллических пленках $\mathrm{Nd}_{2-x} \mathrm{Ce}_{x} \mathrm{CuO}_{4+\delta} / \mathrm{SrTiO}_{3}$ с различной ориентацией оси с относительно плоскости подложки. Было установлено, что коэффициент Холла между проводящими плоскостями $\mathrm{CuO}_{2}$ на два порядка больше, чем коэффициент Холла в плоскостях $\mathrm{CuO}_{2}$, что может быть связано с особенностями некогерентного туннелирования носителей заряда между проводящими плоскостями. 


\section{Список литературы}

[1] H. Takagi, S. Ushida, Y. Tokura. Phys. Rev. Lett. 62, 1197 (1089).

[2] M.V. Sadovskii. Superconductivity and Localization. World Scientific, Singapore (2000). 261 p.

[3] N. Plakida. High-Temperature Cuprate Superconductors, Experiment, Theory and Applications. Springer-Verlag Berlin-Heidelberg (2010). 569 p.

[4] T. Ito, H. Takagi, S. Ishibashi, T. Ido, S. Uchida. Nature 350, 596 (1991).

[5] Т.Б. Чарикова, А.И. Пономарев, Г.И. Харус, Н.Г. Шелушинина, А.О. Ташлыков, А.В. Ткач, А.И. Иванов. ЖЭТФ 132, $712(2007)$.

[6] A.A. Ivanov, S.G. Galkin, A.V. Kuznetsov, A.P. Menushenkov. Physica C 180, 69 (1991).

[7] T.B. Charikova., N.G. Shelushinina, G.I. Harus, D.S. Petukhov, A.I. Ivanov. Solid State Phenomena 215, 77 (2014).

[8] N.G. Shelushinina, A.S. Klepikova, D.S. Petukhov. O.E. Petukhova, T.B. Charikova, A.A. Ivanov. J. Phys.: Conf. Ser. (2018). In press.

[9] A. Cassam-Chenai, D. Maily. Phys. Rev. B 52, 1984 (1995).

[10] R.H. McKenzie, P. Moses. Phys. Rev. Lett. 81, 4492 (1998).

[11] P.W. Anderson. Science 235, 1195 (1987).

[12] T.B. Charikova. N.G. Shelushinina, G.I. Harus, D.S. Petukhov, V.N. Neverov, A.A. Ivanov. Physica C 488, 25 (2013).

[13] Задачи по термодинамике и статистической физике / Под. ред. П. Ландсберга, Мир, М. (1974). гл. 19.

Редактор Т.Н. Василевская 